\title{
Relativistic stabilisation of the diocotron instability in a pulsar "cylindrical" electrosphere
}

\author{
J. Pétri \\ Max-Planck-Institut für Kernphysik, Saupfercheckweg 1, 69117 Heidelberg, Germany \\ e-mail: Jerome.Petri@mpi-hd.mpg.de
}

Received 20 December 2006 / Accepted 13 April 2007

\begin{abstract}
Context. The physics of the pulsar inner magnetosphere remains poorly constrained by observations. Although about 2000 pulsars have been discovered to date, only a little is known about their emission mechanism. Large vacuum gaps exist in the magnetosphere, and a non-neutral plasma partially fills the neutron star surroundings to form an electrosphere.

Aims. In a previous work, we showed that the differentially rotating equatorial disk in the pulsar's electrosphere is diocotron unstable in the non-relativistic regime. In this paper, we extend these results and study the relativistic and electromagnetic stabilisation effects by including the magnetic field perturbation and allowing for relativistic speeds of the guiding centre, in a self-consistent manner. We use the electric drift approximation, valid for low-density plasmas.

Methods. We linearise the coupled relativistic cold-fluid and Maxwell equations in the electric drift approximation. The non-linear eigenvalue problem for the perturbed azimuthal electric field is solved numerically with standard techniques for boundary value problems like the shooting method. The spectrum of the relativistic diocotron instability is computed in a non-neutral plasma column confined between two cylindrically conducting walls.

Results. For low-speed motions, we recover the eigenfunctions and eigenspectra of the non-relativistic diocotron instability. Our algorithm is also checked in the relativistic planar diode geometry for which an analytical expression of the dispersion relation is known. As expected, when the relativistic and electromagnetic effects become significant, the diocotron instability tends to stabilise. In cylindrical geometry, for some special rotation profiles, all azimuthal modes $l$ are completely suppressed for sufficiently relativistic flows. However, for the profile relevant to the electrosphere, depending on the exact rotation curves, the growth rates can either significantly decrease till they vanish or persist for moderate $l$.

Conclusions. The non-neutral plasma flowing in the pulsar electrosphere approaches the speed of light when reaching the lightcylinder. Therefore, relativistic and electromagnetic effects are important. They are able to completely suppress the diocotron instability. Nevertheless, results are sensitive to the tail of the rotation curves; therefore, particle diffusion across the magnetic field due to the diocotron instability only works efficiently close to the neutron star surface.
\end{abstract}

Key words. instabilities - plasmas - magnetohydrodynamics (MHD) - methods: analytical - methods: numerical stars: pulsars: general

\section{Introduction}

The detailed structure of charge distribution and electric-current circulation in the closed magnetosphere of a pulsar remains poorly understood. Although it is often assumed that the plasma fills the space entirely and corotates with the neutron star, it is on the contrary very likely that it only partly fills it, leaving large vacuum gaps between plasma-filled regions. The existence of such gaps in aligned rotators has been very clearly established by Krause-Polstorff \& Michel (1985a,b). Since then, a number of different numerical approaches to the problem have confirmed their conclusions, including some work by Rylov (1989), Shibata (1989), Zachariades (1993), Neukirch (1993), Thielheim \& Wolfsteller (1994), Spitkovsky \& Arons (2002), and by ourselves (Pétri et al. 2002b). This conclusion about the existence of vacuum gaps has been reached from a self-consistent solution of Maxwell's equations in the case of the aligned rotator. Moreover, Smith et al. (2001) have shown by numerical modelling that an initially filled magnetosphere like the GoldreichJulian model evolves by opening up large gaps and stabilises to the partially filled and partially void solution found by KrausePolstorff \& Michel (1985a) and also by Pétri et al. (2002b). The status of models of the pulsar magnetospheres, or electrospheres, has recently been critically reviewed by Michel (2005). A solution with vacuum gaps has the peculiar property that those parts of the magnetosphere that are separated from the star's surface by a vacuum region are not corotating and so suffer differential rotation.

This raises the question of the stability of such a charged plasma flow. The differential rotation in the equatorial, non neutral disk induces the so-called diocotron and magnetron instabilities that are well known to plasma physicists (Oneil 1980; Davidson 1990; O'Neil \& Smith 1992). In the inner parts of the magnetosphere, far from the light cylinder, the instability reduces to its electrostatic form, the diocotron instability. The linear development of the diocotron instability of a thin, differentially rotating charged disk was studied by Pétri et al. (2002a) and Pétri (2007) and shown to proceed at a growth rate comparable to the star's rotation rate. The non linear development of this instability was studied by Pétri et al. (2003) in the framework of an infinitely thin disk model. They have shown that the instability causes a cross-field transport of these charges in the equatorial disk, evolving into a net out-flowing flux of charges. Spitkovsky \& Arons (2002) have numerically studied the problem, and concluded that this charge transport tends to fill the gaps with plasma. The appearance of a cross-field electric 
current as a result of the diocotron instability has been observed by Pasquini \& Fajans (2002) in laboratory experiments in which charged particles were continuously injected in the plasma column trapped in a Malmberg-Penning configuration.

The aim of this work is to extend the previous work in Pétri (2007) on the diocotron instability by including electromagnetic and relativistic effects. Indeed, when the plasma approaches the light cylinder, the guiding centre motion becomes relativistic and magnetic perturbations become significant. The relativistic aspect of the diocotron instability has already been investigated in the planar diode geometry by Davidson et al. $(1987,1988)$. They clearly demonstrate the stabilisation due to electromagnetic effects. Applications to the planar magnetron geometry was investigated by Ayres et al. (1992).

In this paper we present a numerical analysis of the linear growth rate of the relativistic diocotron instability for a plasma column. The paper is organised as follows. In Sect. 2, we describe the initial setup of the plasma column consisting of an axially symmetric equilibrium between two conducting walls. We give several equilibrium profiles useful for studying the diocotron instability in different configurations. In Sect. 3, the nonlinear eigenvalue problem satisfied by the perturbed azimuthal electric field component is derived. The algorithm to solve the eigenvalue problem is checked against known analytical results in the non-relativistic cylindrical limit, as well as in the relativistic planar diode geometry, Sect. 4. Then, applications to some typical equilibrium configurations are shown in Sect. 5. First we consider a plasma column with constant diocotron frequency. Next, we study the effect of the cylindrical geometry (curvature of the flow) and the transition to the planar diode limit. Finally, the stabilisation of the diocotron instability in a pulsar electrosphere is demonstrated when the flow becomes ultra-relativistic. The conclusions and the possible generalisation are presented in Sect. 6.

\section{Initial setup}

We study the motion of a non neutral plasma column of infinite axial extent along the $z$-axis in the electric drift approximation. This approximation is well-suited to low-density plasmas satisfying $\omega_{\mathrm{p}}^{2} \ll \omega_{\mathrm{c}}^{2}$, where $\omega_{\mathrm{p}}$ is the plasma frequency and $\omega_{\mathrm{c}}$ the cyclotron frequency. The geometric configuration is the same as in Pétri (2007). However, perturbations in the magnetic field induced by the relativistic motion of the charges are now taken into account in a fully self-consistent manner. Therefore, we have to solve the full set of Maxwell equations.

In this section, we briefly summarise the equilibrium conditions imposed on the plasma and give some typical examples of equilibrium configurations for specified velocity, density, and electric field profiles.

\subsection{Equilibrium of the plasma column}

We consider a single-species non-neutral plasma consisting of particles with mass $m_{\mathrm{e}}$ and charge $q$ trapped between two cylindrically conducting walls located at $r=W_{1}$ and $r=W_{2}>W_{1}$. The plasma column itself is confined between $R_{1} \geq W_{1}$ and $R_{2} \leq W_{2}$. This allows us to take vacuum regions between the plasma and the conducting walls into account. We adopt cylindrical coordinates denoted by $(r, \varphi, z)$ and the corresponding basis vectors $\left(\boldsymbol{e}_{r}, \boldsymbol{e}_{\varphi}, \boldsymbol{e}_{z}\right)$.

In the equilibrium configuration, the particle number density is $n_{\mathrm{e}}(r)$ and the associated charge density is $\rho_{\mathrm{e}}(r)=q n_{\mathrm{e}}(r)$.
Particles evolve in a cross electric and magnetic field such that the equilibrium magnetic field is directed along the $z$-axis, whereas the equilibrium electric field is directed along the $r$-axis. The electric field induced by the plasma itself, $\boldsymbol{E}_{\mathrm{p}}$, is

$\boldsymbol{E}_{\mathrm{p}}=E_{r} \boldsymbol{e}_{r}$

The magnetic field is made up of two parts, the first an imposed external applied field, $\boldsymbol{B}_{0}$, assumed to be uniform in the region outside the plasma column, and the second a plasma induced field, $\boldsymbol{B}_{\mathrm{p}}$

$\boldsymbol{B}=\boldsymbol{B}_{\mathrm{p}}+\boldsymbol{B}_{0}=B_{z} \boldsymbol{e}_{z}$

Therefore, for azimuthally symmetric equilibria, the steady-state Maxwell-Gauss and Maxwell-Ampère equations satisfy

$$
\begin{aligned}
\frac{1}{r} \frac{\partial}{\partial r}\left(r E_{r}\right) & =\frac{\rho_{\mathrm{e}}}{\varepsilon_{0}} \\
\frac{\partial B_{z}}{\partial r} & =-\mu_{0} \rho_{\mathrm{e}} v_{\varphi}
\end{aligned}
$$

In the electric drift approximation, particle trajectories are described by the guiding-centre motion, so that the flow velocity is only azimuthal and expressed as

$$
\boldsymbol{v}=v_{\varphi} \boldsymbol{e}_{\varphi}=-\frac{E_{r}}{B_{z}} \boldsymbol{e}_{\varphi}
$$

In order to avoid unphysical superluminal motion of the guiding centre, $v<c$, this model can only be applied for $E_{r}<c B_{z}$. In the opposite case, i.e. when $E_{r}>c B_{z}$, inertia effects become significant and should be included in the equation of motion. The most general instability in a non-neutral plasma, when taking inertia and relativistic effects into account, is the so-called magnetron instability. The full self-consistent response of the plasma to this instability will be studied in a forthcoming paper (removing the electric drift approximation, replaced by the relativistic momentum equation). In the case of pulsar magnetospheres, let us estimate the distance to which this approximation remains valid. The drift motion, Eq. (5), applies roughly when the Larmor radius of the particles $R_{\mathrm{g}}$ is less than the radius of their orbit $R$. Assuming relativistic speeds, with a guiding-centre motion at the star rotation speed $\Omega_{*}$, we get

$R_{\mathrm{g}}=\frac{\gamma m_{\mathrm{e}} c}{|q| B}=\frac{m_{\mathrm{e}} c}{|q| B \sqrt{1-R^{2} \Omega_{*}^{2} / c^{2}}}<R$

Let $R_{*}$ and $B_{*}$ be the radius of the neutron star and the surface magnetic field, respectively. For a dipolar vacuum magnetic field, we have

$$
B(R)=B_{*} \frac{R_{*}^{3}}{R^{3}} .
$$

Condition Eq. (6) can be cast into

$\frac{R^{2}}{\sqrt{1-R^{2} \Omega_{*}^{2} / c^{2}}}<\frac{|q| B_{*} R_{*}^{3}}{m_{\mathrm{e}} c} \equiv Y^{2}$.

We introduce the parameter

$$
Y=\sqrt{\frac{|q| B_{*} R_{*}^{3}}{m_{\mathrm{e}} c}}
$$


Thus, we find that a rough estimate of the largest radius where the drift approximation remains valid is

$$
\frac{R_{\max }}{R_{\mathrm{L}}} \lesssim \frac{Y^{2}}{\sqrt{2} R_{\mathrm{L}}^{2}} \sqrt{\sqrt{1+4 \frac{R_{\mathrm{L}}^{4}}{Y^{4}}}-1}
$$

Applying to a pulsar, with a period of the order of one second, $P=1 \mathrm{~s}$ and magnetic fields of the order $B_{*}=10^{8} \mathrm{~T}$, and assuming a typical neutron star radius of $R_{*}=10 \mathrm{~km}$, we get

$R_{\mathrm{L}}=4771 R_{*} ; \quad Y=5076 R_{\mathrm{L}} \gg R_{\mathrm{L}}$.

Thus $R_{\max } \lesssim R_{\mathrm{L}}$. The approximation fails only very close to the light cylinder $R_{\mathrm{L}}$. For millisecond pulsars, $P=1 \mathrm{~ms}$ and $B_{*}=10^{5} \mathrm{~T}$, the same conclusion applies because

$R_{\mathrm{L}}=4.771 R_{*} ; \quad Y=160531 R_{\mathrm{L}} \gg R_{\mathrm{L}}$.

We conclude that the electric-drift approximation remains valid to good accuracy for electrospheric plasmas confined within the light cylinder.

For a constant density profile in the plasma column with $W_{1}=0$, the electric drift approximation, Eq. (5), corresponds to a circular motion at the diocotron frequency defined by (see for instance Davidson 1990)

$\omega_{\mathrm{D}}=\frac{\omega_{\mathrm{p}}^{2}}{2 \omega_{\mathrm{c}}}$

The relativistic plasma and cyclotron frequencies are given respectively by

$$
\begin{aligned}
\omega_{\mathrm{p}}^{2} & =\frac{\rho_{\mathrm{e}} q}{\gamma m_{\mathrm{e}} \varepsilon_{0}} \\
\omega_{\mathrm{c}} & =\frac{q B_{z}}{\gamma m_{\mathrm{e}}}
\end{aligned}
$$

where $\gamma=1 / \sqrt{1-v_{\varphi}^{2} / c^{2}}$ corresponds to the bulk Lorentz factor of the flow.

We assume that the electric field induced by the plasma vanishes at the inner wall, at $r=W_{1}$, i.e.

$\boldsymbol{E}_{\mathrm{p}}\left(W_{1}\right)=\mathbf{0}$.

Integrating Eq. (3) therefore gives for the electric field generated by the plasma

$\boldsymbol{E}_{\mathrm{p}}(r)=\frac{1}{\varepsilon_{0} r} \int_{W_{1}}^{r} \rho_{\mathrm{e}}\left(r^{\prime}\right) r^{\prime} \mathrm{d} r^{\prime} \boldsymbol{e}_{\mathrm{r}}$.

For the magnetic field induced by the plasma, we solve Eq. (4) with the boundary condition $B_{z}\left(R_{2}\right)=B_{0}$. This simply states that the total magnetic field outside the plasma column has to match the magnetic field imposed by an external device.

Any equilibrium state is completely determined by the following four quantities: the total radial electric field, $E_{r}$, the total axial magnetic field, $B_{z}$, the charge density, $\rho_{\mathrm{e}}$, and the azimuthal speed of the guiding centre, $v_{\varphi}$. Prescribing one of these profiles, the remaining three are found self-consistently by solving the set of Eqs. (3)-(5). We give some typical examples in the next sections in which the velocity profile, the density profile, or the electric field is imposed.

\subsection{Specified velocity profile}

Let us first assume that the velocity profile $v_{\varphi}=r \Omega$ is prescribed. This case is well-suited for the study of the pulsar's electrosphere in which the plasma is in differential rotation. Although the magnetic field is probably nearly dipolar in a pulsar, we assume a uniform magnetic field in a vacuum in order to remain fully self-consistent. We know from the previous analysis (Pétri 2007) that the growth rates are not very sensitive to the magnetic field structure. As already noticed in this work, the differential rotation is essential to the presence of the diocotron instability. The other equilibrium quantities, $\left(E_{r}, B_{z}, \rho_{\mathrm{e}}\right)$, are easily derived from $\Omega$. Indeed, inserting $\rho_{\mathrm{e}}$ from Eq. (3) and $E_{r}$ from Eq. (5) into the Maxwell-Ampère Eq. (4), the magnetic field satisfies a first-order, ordinary linear differential equation

$$
\frac{\partial B_{z}}{\partial r}=\frac{\gamma^{2} \beta}{r} B_{z} \frac{\partial}{\partial r}(r \beta)=\frac{\gamma^{2} \Omega}{c^{2}} B_{z} \frac{\partial}{\partial r}\left(r^{2} \Omega\right) .
$$

The Lorentz factor of the flow is

$$
\begin{aligned}
& \gamma=\frac{1}{\sqrt{1-\beta^{2}}} \\
& \beta=\frac{r \Omega}{c} .
\end{aligned}
$$

From the Poisson equation, Eq. (3), and the definition of the plasma frequencies, Eqs. (14) and (15), the charge density is found by

$\frac{\rho_{\mathrm{e}}}{\varepsilon_{0} B_{z}}=\frac{\omega_{\mathrm{p}}^{2}}{\omega_{\mathrm{c}}}=-\frac{\gamma^{2}}{r} \frac{\partial}{\partial r}\left(r^{2} \Omega\right)=-\gamma^{2}\left[2 \Omega+r \frac{\partial \Omega}{\partial r}\right]$.

The electric field is recovered from the Poisson equation, Eq. (3), or, according to the electric drift approximation, by Eq. (5) leading to the charge density

$\rho_{\mathrm{e}}=-\varepsilon_{0}\left[\frac{B_{z}}{r} \frac{\partial}{\partial r}\left(r^{2} \Omega\right)+r \Omega \frac{\partial B_{z}}{\partial r}\right]$

consistent with Eqs. (18) and (21).

\subsection{Specified density profile}

A simple and useful charge-density profile for studying the relativistic effect on the diocotron instability is given by a constant diocotron frequency in the whole plasma column, Eq. (13), such that

$\omega_{\mathrm{D}}(r)= \begin{cases}0, & W_{1} \leq r \leq R_{1} \\ \omega_{\mathrm{D}}=\text { const. }, & R_{1} \leq r \leq R_{2} \\ 0, & R_{2} \leq r \leq W_{2}\end{cases}$

Using Eq. (21), the rotation profile is deduced by integrating an ordinary differential equation for $\Omega$,

$$
\frac{\partial \Omega}{\partial r}=-\frac{2}{r}\left[\Omega+\omega_{\mathrm{D}}\left(1-\frac{r^{2} \Omega^{2}}{c^{2}}\right)\right]
$$

with the boundary condition that $\Omega$ vanishes at the inner plasma edge, $\Omega\left(R_{1}\right)=0$, to insure consistency with Eq. (16). Knowing $\Omega$, the same procedure as in the previous subsection for a specified velocity profile is applied; i.e. the magnetic field is calculated according to Eq. (18). 


\subsection{Specified electric field}

It is also possible to specify the equilibrium radial electric field. An interesting case is given by

$E_{r}(r)= \begin{cases}0, & W_{1} \leq r \leq R_{1} \\ -B_{0} \frac{\sinh \alpha\left(r-R_{1}\right)}{\cosh \alpha\left(R_{2}-R_{1}\right)}, & R_{1} \leq r \leq R_{2}, \\ -B_{0} \frac{R_{2} \tanh \alpha\left(R_{2}-R_{1}\right)}{r}, & R_{2} \leq r \leq W_{2}\end{cases}$

where $\alpha$ is a constant useful to adjust the maximal speed of the column at $R_{2}$

$v_{\max }=\tanh \alpha\left(R_{2}-R_{1}\right)$.

The equilibrium electric profile, Eq. (25), enables us to investigate the influence of the cylindrical geometry compared to the planar diode geometry. Indeed, in the limit of small curvature of the column, i.e. when $R_{2}-R_{1} \ll R_{1}$, the eigenvalue problem in cylindrical geometry reduces to the planar diode case. The charge density is found by Eq. (3), whereas the magnetic field is the solution of an ordinary differential equation

$\frac{\partial B_{z}^{2}}{\partial r}=\frac{1}{r^{2} c^{2}} \frac{\partial}{\partial r}\left(r^{2} E_{r}^{2}\right)$.

Finally, the velocity is given by the electric drift approximation, Eq. (5).

\section{Linear analysis}

In this section, we show how to derive the eigenvalue problem for the diocotron instability in the relativistic regime. Let us start with the full set of non-linear equations governing the plasma.

\subsection{Equations of motion}

Each particle evolves in the self-consistent electromagnetic field partly imposed by an external device and partly induced by the plasma itself. The motion of the plasma column is governed by the conservation of charge, the electric drift approximation, and the full set of Maxwell equations, respectively,

$$
\begin{aligned}
\frac{\partial \rho_{\mathrm{e}}}{\partial t}+\operatorname{div}\left(\rho_{\mathrm{e}} \boldsymbol{v}\right) & =0 \\
\boldsymbol{v} & =\frac{\boldsymbol{E} \wedge \boldsymbol{B}}{B^{2}} \\
\operatorname{rot} \boldsymbol{E} & =-\frac{\partial \boldsymbol{B}}{\partial t} \\
\operatorname{rot} \boldsymbol{B} & =\mu_{0} \boldsymbol{j}+\varepsilon_{0} \mu_{0} \frac{\partial \boldsymbol{E}}{\partial t} \\
\operatorname{div} \boldsymbol{E} & =\frac{\rho_{\mathrm{e}}}{\varepsilon_{0}} \\
\operatorname{div} \boldsymbol{B} & =0 .
\end{aligned}
$$

For a non-neutral plasma, the current density is related to the charge density by

$\boldsymbol{j}=\rho_{\mathrm{e}} \boldsymbol{v}$.

We apply the standard linear perturbation theory. All perturbations of physical quantities $X$, like electric field, density, and velocity components, are expressed by the expansion

$X(r, \varphi, t)=X(r) \mathrm{e}^{i(l \varphi-\omega t)}$, where $l$ is the azimuthal mode and $\omega$ the eigenfrequency. Therefore, we do not take the finite thickness of the disk into account. The whole cylinder moves as one block in the vertical direction. However, because the disk of the pulsar electrosphere has a finite vertical dimension, denoted by $H$, these modes should also depend on the vertical coordinate $z$. It is possible to extend this cylindrical model to modes depending on $z$ by introducing the following expansion

$X(r, \varphi, z, t)=X(r) \mathrm{e}^{\mathrm{i}(k z+l \varphi-\omega t)}$.

The technique is similar to the one used to investigate wave propagation in accretion disks of finite thickness. Several methods could be applied. For instance, Nowak \& Wagoner (1991) used a series expansion in $z / H$ of the Lagrangian displacement of a fluid element in order to study trapped adiabatic oscillations in accretion disks. Looking for solutions that are separable in the radial and vertical directions is another means of seeking the perturbations. This was done, for instance, by Okazaki et al. (1987) for trapped oscillations in relativistic accretion disks and by Kato (1989) for low-frequency corrugation waves in an isothermal relativistic accretion disk. For insight into the properties of the oscillations, Perez et al. (1997) performed a radial and vertical WKB analysis.

In our case, for a small vertical extension of the disk, $k H \gg$ 1 , a series expansion in $z / H$ would be appropriate. Indeed, regions with $z \gg H$ will not significantly contribute to the electromagnetic field because of the oscillating term $\mathrm{e}^{\mathrm{i} k z}$. However, this more general study is left for future work.

\subsection{Linearisation}

We study the stability of the plasma column around the equilibrium mentioned in the previous section. An expansion to the first order for the electromagnetic field around the equilibrium $\left(\boldsymbol{E}^{0}, B_{z}^{0}\right)$ leads us to

$\boldsymbol{E}=\boldsymbol{E}^{0}+\delta \boldsymbol{E}$

$B_{z}=B_{z}^{0}+\delta B_{z}$

and the same for the charge and current density

$$
\boldsymbol{j}=\boldsymbol{j}^{0}+\delta \boldsymbol{j}
$$$$
\rho_{\mathrm{e}}=\rho_{\mathrm{e}}^{0}+\delta \rho_{\mathrm{e}} \text {. }
$$

Linearising the set of Maxwell equations, we have

$$
\begin{aligned}
\frac{1}{r} \frac{\partial}{\partial r}\left(r \delta E_{r}\right)+\mathrm{i} \frac{l}{r} \delta E_{\varphi} & =\frac{\delta \rho_{\mathrm{e}}}{\varepsilon_{0}} \\
\frac{1}{r} \frac{\partial}{\partial r}\left(r \delta E_{\varphi}\right)-\mathrm{i} \frac{l}{r} \delta E_{r} & =\mathrm{i} \omega \delta B_{z} \\
\mathrm{i} \frac{l}{r} \delta B_{z} & =\mu_{0} \delta j_{\mathrm{r}}-\mathrm{i} \frac{\omega}{c^{2}} \delta E_{r} \\
-\frac{\partial}{\partial r} \delta B_{z} & =\mu_{0} \delta j_{\varphi}-\mathrm{i} \frac{\omega}{c^{2}} \delta E_{\varphi} .
\end{aligned}
$$

The current density perturbation is

$$
\begin{aligned}
\delta j_{r} & =\rho_{\mathrm{e}} \delta v_{r} \\
\delta j_{\varphi} & =\delta \rho_{\mathrm{e}} v_{\varphi}+\rho_{\mathrm{e}} \delta v_{\varphi} .
\end{aligned}
$$

It is convenient to introduce a new function $\phi$ related to the azimuthal electric field by $\delta E_{\varphi}=-\mathrm{i} l \phi / r$ (we emphasise that this function is not the scalar potential from which the electric field 
could be derived, but is just a convenient auxiliary variable) such that the electric and magnetic fields become

$\delta E_{r}=-\kappa(r, \omega)\left[\frac{\partial \phi}{\partial r}-\mathrm{i} \mu_{0} \omega \frac{r^{2}}{l^{2}} \rho_{\mathrm{e}} \delta v_{r}\right]$
$\delta B_{z}=\frac{\omega r}{l} \kappa(r, \omega)\left[\frac{1}{c^{2}} \frac{\partial \phi}{\partial r}-\mathrm{i} \frac{\mu_{0}}{\omega} \rho_{\mathrm{e}} \delta v_{r}\right]$,

where we introduced the function

$\kappa(r, \omega)=\frac{1}{1-(\omega r / l c)^{2}}$.

The Maxwell-Gauss Eq. (32), is therefore written

$$
\begin{aligned}
& \frac{1}{r} \frac{\partial}{\partial r}\left(r \kappa(r, \omega) \frac{\partial \phi}{\partial r}\right)-\frac{l^{2}}{r^{2}} \phi= \\
& \quad-\frac{\delta \rho_{\mathrm{e}}}{\varepsilon_{0}}+\mathrm{i} \mu_{0} \frac{\omega}{r} \frac{\partial}{\partial r}\left(\frac{r^{3}}{l^{2}} \kappa(r, \omega) \rho_{\mathrm{e}} \delta v_{r}\right) .
\end{aligned}
$$

From the continuity Eq. (28), we get

$\delta \rho_{\mathrm{e}}=\frac{1}{\mathrm{i}(\omega-l \Omega)}\left[\frac{1}{r} \frac{\partial}{\partial r}\left(r \rho_{\mathrm{e}} \delta v_{r}\right)+\mathrm{i} \frac{l}{r} \rho_{\mathrm{e}} \delta v_{\varphi}\right]$.

The electric drift approximation gives

$$
\begin{aligned}
& \delta v_{r}=\frac{\delta E_{\varphi}}{B_{z}}=-\mathrm{i} \frac{l}{r} \frac{\phi}{B_{z}} \\
& \delta v_{\varphi}=\frac{E_{r} \delta B_{z}-\delta E_{r} B_{z}}{B_{z}^{2}} .
\end{aligned}
$$

After some algebra, the eigenvalue problem for the relativistic diocotron instability in cylindrical geometry is written

$$
\begin{aligned}
\frac{1}{r} \frac{\partial}{\partial r} & {\left[r \kappa(r, \omega) \frac{\partial \phi}{\partial r}\right]-\frac{l^{2}}{r^{2}}[1+\chi(r, \omega)] \phi=} \\
& \frac{l \phi}{r(\omega-l \Omega)} \kappa(r, \omega)\left[1-\frac{\Omega r}{c} \frac{\omega r}{l c}\right] \frac{\partial}{\partial r}\left(\frac{\omega_{\mathrm{p}}^{2}}{\omega_{\mathrm{c}}}\right)
\end{aligned}
$$

with

$\chi(r, \omega)=\kappa(r, \omega) \frac{\omega_{\mathrm{p}}^{2}}{\omega_{\mathrm{c}}}\left(\frac{r}{l c}\right)^{2}\left(\frac{\omega_{\mathrm{p}}^{2}}{\omega_{\mathrm{c}}}+2 \kappa(r, \omega) \frac{\omega}{l}\right)$.

The eigenvalue Eq. (54) is very general. It describes the motion of small electromagnetic perturbations around the given equilibrium state, Eqs. (3) and (4), in the electric drift approximation, Eq. (5). Many aspects of the relativistic diocotron instability can be investigated with this eigenvalue equation. In order to solve the eigenvalue problem, boundary conditions need to be imposed at the plasma/vacuum interface. They play a decisive role in the presence or absence of the instability. How to treat these transitions between plasma and vacuum is discussed in the next subsection.

Note that in the non-relativistic limit, the generalised linear eigenvalue problem, Eq. (20) in Pétri (2007), is recovered:

$\frac{1}{r} \frac{\partial}{\partial r}\left[r \frac{\partial \phi}{\partial r}\right]-\frac{l^{2}}{r^{2}} \phi=\frac{l \phi}{r(\omega-l \Omega)} \frac{\partial}{\partial r}\left(\frac{\omega_{\mathrm{p}}^{2}}{\omega_{\mathrm{c}}}\right)$.

This is the standard eigenvalue problem for the non-relativistic diocotron instability in cylindrical geometry.

\subsection{Boundary conditions}

In laboratory experiments, the plasma is usually confined between inner and outer conducting walls. However, in pulsar electrospheres, no such outer device exists to constrain the electric field at the outer boundary. Radiation from the plasma could propagate into vacuum to infinity, carrying energy away from the plasma by Poynting flux. To allow for this electromagnetic wave production by the instabilities studied in this work, the outer wall is removed. The electromagnetic field is solved analytically in vacuum and matched to the solution in the plasma at the plasma/vacuum interface located at $r=R_{2}$. First we discuss the situation in which an outer wall exists and then consider outgoing waves.

\subsubsection{Outer wall}

When vacuum regions exist between the plasma column and the walls, special care is required at the sharp plasma/vacuum interfaces. Indeed, the right-hand side of Eq. (54) then involves Dirac distribution functions $\delta(r)$ because the function $\omega_{\mathrm{p}}^{2} / \omega_{\mathrm{c}}$ is discontinuous at $R_{1}$ and $R_{2}$. In other words, its derivative is

$$
\begin{aligned}
\frac{\partial}{\partial r}\left(\frac{\omega_{\mathrm{p}}^{2}}{\omega_{\mathrm{c}}}\right)= & \left.\delta\left(r-R_{1}\right) \frac{\partial}{\partial r}\left(\frac{\omega_{\mathrm{p}}^{2}}{\omega_{\mathrm{c}}}\right)\right|_{r=R_{1}} \\
& -\left.\delta\left(r-R_{2}\right) \frac{\partial}{\partial r}\left(\frac{\omega_{\mathrm{p}}^{2}}{\omega_{\mathrm{c}}}\right)\right|_{r=R_{2}}+\left.\frac{\partial}{\partial r}\left(\frac{\omega_{\mathrm{p}}^{2}}{\omega_{\mathrm{c}}}\right)\right|_{\text {regular }}
\end{aligned}
$$

where $\|_{\text {regular }}$ means the regular (or continuous) part of the derivative; i.e. it does not involve distribution functions. It vanishes in the vacuum regions, $r<R_{1}$ and $r>R_{2}$. Therefore, the first order derivative of $\phi$ is not continuous at these interfaces. To overcome this difficulty, we decompose the space between the two walls into three distinct regions:

- region I: vacuum space between inner wall and inner boundary of the plasma column, with the solution for the function $\phi$ denoted by $\phi_{\mathrm{I}}$, defined for $W_{1} \leq r \leq R_{1}$;

- region II: the plasma column itself, located between $R_{1}$ and $R_{2}$, with the solution denoted by $\phi_{\mathrm{II}}$ and defined for $R_{1} \leq r \leq$ $R_{2}$

- region III: vacuum space between the outer boundary of the plasma column and the outer wall, solution denoted by $\phi_{\mathrm{III}}$, defined for $R_{2} \leq r \leq W_{2}$.

In regions I and III, the vacuum solutions should satisfy the required boundary conditions, $\phi_{\mathrm{I}}\left(W_{1}\right)=0$ and $\phi_{\mathrm{III}}\left(W_{2}\right)=0$.

The jumps in the derivative $\partial \phi / \partial r$ at each interface are easily found from Eq. (57). At $R_{1}$, the jump is

$$
\begin{aligned}
\frac{\partial \phi_{\mathrm{II}}}{\partial r}\left(R_{1}\right)-\frac{\partial \phi_{\mathrm{I}}}{\partial r}\left(R_{1}\right)= & \frac{l \phi\left(R_{1}\right)}{R_{1}\left(\omega-l \Omega\left(R_{1}\right)\right)} \\
& \times\left(1-\frac{\Omega\left(R_{1}\right) R_{1}}{c} \frac{\omega R_{1}}{l c}\right) \frac{\omega_{\mathrm{p}}^{2}\left(R_{1}\right)}{\omega_{\mathrm{c}}\left(R_{1}\right)}
\end{aligned}
$$

Similarly, at the outer interface at $R_{2}$, we obtain,

$$
\begin{aligned}
\frac{\partial \phi_{\mathrm{III}}}{\partial r}\left(R_{2}\right)-\frac{\partial \phi_{\mathrm{II}}}{\partial r}\left(R_{2}\right)= & -\frac{l \phi\left(R_{2}\right)}{R_{2}\left(\omega-l \Omega\left(R_{2}\right)\right)} \\
& \times\left(1-\frac{\Omega\left(R_{2}\right) R_{2}}{c} \frac{\omega R_{2}}{l c}\right) \frac{\omega_{\mathrm{p}}^{2}\left(R_{2}\right)}{\omega_{\mathrm{c}}\left(R_{2}\right)}
\end{aligned}
$$




\subsubsection{Outgoing wave solution}

Because of the wall located at $r=W_{2}$, the outer boundary condition $\phi_{\text {III }}\left(W_{2}\right)=0$ enforces $E_{\varphi}\left(W_{2}\right)=0$. It therefore prevents waves escaping from the system due to the vanishing outgoing Poynting flux, $E_{\varphi} B_{z} / \mu_{0}=0$. In pulsar magnetospheres, no such wall exists. In order to let the system produce outgoing electromagnetic waves, we thus remove the outer wall in this case and solve the vacuum wave equation for $\phi$, which then reads

$\frac{1}{r} \frac{\partial}{\partial r}\left[r \kappa(r, \omega) \frac{\partial \phi}{\partial r}\right]-\frac{l^{2}}{r^{2}} \phi=0$.

This equation can also be derived directly from the vector wave equation

$\Delta \boldsymbol{E}-\frac{1}{c^{2}} \frac{\partial^{2} \boldsymbol{E}}{\partial t^{2}}=\mathbf{0}$

projected along the $e_{\varphi}$ axis. To find the right outgoing wave boundary conditions, it is therefore necessary to solve the vectorwave equation in cylindrical coordinates using vector cylindrical harmonics as described for instance in Stratton (1941) and Morse \& Feshbach (1953). The solutions for the function $\phi$ to be an outgoing wave in a vacuum outside the plasma column, which vanishes at infinity, is given by (region III with $W_{2}=+\infty$ )

$\phi_{\mathrm{III}}=K r \frac{\partial}{\partial r} H_{l}\left(\frac{\omega r}{c}\right)=K \frac{\omega r}{c} H_{l}^{\prime}\left(\frac{\omega r}{c}\right)$,

where the cylindrical outgoing wave function is given by $H_{l}$ (Stratton 1941), the Hankel function of the first kind and of order $l$ related to the Bessel functions by $H_{l}(x)=J_{l}(x)+\mathrm{i} Y_{l}(x)$, (Abramowitz \& Stegun 1965). The prime' means derivative of the function evaluated at the point given in parentheses, and $K$ is a constant to be determined from the boundary condition at $R_{2}$. Eliminating the constant $K$, we conclude that the boundary condition to impose on $\phi$ is

$$
\begin{aligned}
{\left[H_{l}^{\prime}\left(\frac{\omega R_{2}}{c}\right)+\right.} & \left.\frac{\omega R_{2}}{c} H_{l}^{\prime \prime}\left(\frac{\omega R_{2}}{c}\right)\right] \phi_{\mathrm{III}}\left(R_{2}\right) \\
& -R_{2} H_{l}^{\prime}\left(\frac{\omega R_{2}}{c}\right) \frac{\partial \phi_{\mathrm{III}}}{\partial r}\left(R_{2}\right)=0 .
\end{aligned}
$$

The boundary conditions expressed in region II for $\phi_{\mathrm{II}}$ are found by replacing $\phi_{\mathrm{III}}^{\prime}\left(R_{2}\right)$ from Eq. (59). Since $\phi$ is continuous, $\phi_{\mathrm{III}}\left(R_{2}\right)=\phi_{\mathrm{II}}\left(R_{2}\right)=\phi\left(R_{2}\right)$. We find

$$
\begin{aligned}
& {\left[H_{l}^{\prime}\left(\frac{\omega R_{2}}{c}\right)+\frac{\omega R_{2}}{c} H_{l}^{\prime \prime}\left(\frac{\omega R_{2}}{c}\right)\right] \phi\left(R_{2}\right)} \\
& -R_{2} H_{l}^{\prime}\left(\frac{\omega R_{2}}{c}\right)\left[\frac{\partial \phi_{\mathrm{II}}}{\partial r}\left(R_{2}\right)\right. \\
& \left.-\frac{l \phi\left(R_{2}\right)}{R_{2}\left(\omega-l \Omega\left(R_{2}\right)\right)}\left(1-\frac{\Omega\left(R_{2}\right) R_{2}}{c} \frac{\omega R_{2}}{l c}\right) \frac{\omega_{\mathrm{p}}^{2}\left(R_{2}\right)}{\omega_{\mathrm{c}}\left(R_{2}\right)}\right]=0 .
\end{aligned}
$$

\subsection{Algorithm}

The eigenvalue problem, Eq. (54), is solved by standard numerical techniques. We have implemented a shooting method as follows.

First, we guess a good initial value for the eigenvalue $\omega$. Then, the ordinary differential equation, Eq. (54), is integrated numerically from $W_{1}$ to $W_{2}$ with a fifth-order Runge-Kutta or a Bulirsch-Stoer method. More precisely, at $r=W_{1}$, the initial conditions are $\phi=0$ and $\partial \phi / \partial r=1$. Integration is performed in region I until the first vacuum/plasma interface is reached at $r=R_{1}$. There, the first-order derivative in $\phi$ is subject to a discontinuity given by the jump in Eq. (58). Knowing $\partial \phi_{\mathrm{I}} / \partial r\left(R_{1}\right)$, we deduce $\partial \phi_{\mathrm{II}} / \partial r\left(R_{1}\right)$. Therefore, the integration is continued in region II with the initial conditions, $\phi_{\mathrm{II}}\left(R_{1}\right)=\phi_{\mathrm{I}}\left(R_{1}\right)$ (because $\phi$ is continuous) and $\partial \phi_{\text {II }} / \partial r\left(R_{2}\right)$ until the second vacuum/plasma interface is reached at $R_{2}$. The first-order derivative in $\phi$ is now subject to another discontinuity given by the jump in Eq. (59). Knowing $\partial \phi_{\mathrm{II}} / \partial r\left(R_{2}\right)$, we deduce $\partial \phi_{\mathrm{III}} / \partial r\left(R_{2}\right)$. Integration is continued in region III with the initial conditions, $\phi_{\mathrm{III}}\left(R_{2}\right)=\phi_{\mathrm{II}}\left(R_{2}\right)$ and $\partial \phi_{\mathrm{III}} / \partial r\left(R_{2}\right)$, until the endpoint $W_{2}$. At the end of the process, the function $\phi_{\mathrm{III}}$ does not necessarily satisfy the desired boundary conditions. Indeed, the eigenvalue is found whenever the function $\phi$ at the outer wall vanishes $\phi_{\mathrm{III}}\left(W_{2}\right)=0$. Finding $\omega$ is therefore equivalent to finding the root of $\phi_{\mathrm{III}}\left(W_{2}\right)$ with respect to the eigenvalue $\omega$.

For the pulsar electrosphere, the situation is very similar, except that no calculation is performed in region III. The boundary condition for outgoing waves is applied at $R_{2}$, see Eq. (64). Actually for pulsars, we compare both boundary conditions.

\section{Algorithm check}

To check our algorithm in different configurations, we computed the eigenvalues for both a non-relativistic cylindrical plasma column and a relativistic planar diode geometry. For some special density profiles, the exact analytical dispersion relations are known and used for comparison with the numerical results.

\subsection{Non-relativistic plasma column}

In cylindrical geometry, an exact analytical solution for the dispersion relation can be found in the non-relativistic case, (Davidson 1990). We used these results to check our algorithm in cylindrical coordinates.

The magnetic field is constant and uniform in the whole space, $B_{z}(r)=B_{0}$. We do not need to solve the Maxwell-Ampère equation because the magnetic perturbation is neglected in the non-relativistic limit. The particle number density and charge density are also uniform in the whole plasma column such that

$\rho_{\mathrm{e}}(r)= \begin{cases}0, & W_{1} \leq r \leq R_{1} \\ \rho_{0}=\text { const., } & R_{1} \leq r \leq R_{2} . \\ 0, & R_{2} \leq r \leq W_{2}\end{cases}$

Solving the Maxwell-Gauss equation (32), the equilibrium radial electric field is

$$
E_{r}(r)= \begin{cases}0, & W_{1} \leq r \leq R_{1} \\ \frac{\rho_{0} r}{2 \varepsilon_{0}}\left(1-\frac{R_{1}^{2}}{r^{2}}\right), & R_{1} \leq r \leq R_{2}, \\ \frac{\rho_{0}}{2 \varepsilon_{0}} \frac{R_{2}^{2}-R_{1}^{2}}{r}, & R_{2} \leq r \leq W_{2}\end{cases}
$$

and the corresponding electric drift speed in the plasma

$\Omega=-\omega_{\mathrm{D}}\left(1-\frac{R_{1}^{2}}{r^{2}}\right)$

The diocotron frequency is constant, $\omega_{\mathrm{D}}=\rho_{0} / 2 \varepsilon_{0} B_{0}=$ const. The solutions to the dispersion relation for this particular case are

$$
\omega=-\frac{\omega_{\mathrm{D}}}{2}\left[b_{l} \pm \sqrt{b_{l}^{2}-4 c_{l}}\right]
$$


Table 1. Numerical eigenvalues $\omega_{\text {num }}$ and relative errors for the non-relativistic plasma column for different mode $l$ and different aspect ratios, $d_{1}=R_{1} / W_{2}$, and $d_{2}=R_{2} / W_{2}$ with $W_{1}=1$ and $W_{2}=10$. The precision of the computed eigenvalues reaches 10 digits.

\begin{tabular}{|c|c|c|c|c|c|}
\hline mode $l$ & $d_{1}$ & $d_{2}$ & $\omega_{\text {num }}$ & $\varepsilon_{\mathrm{Re}}$ & $\varepsilon_{\mathrm{Im}}$ \\
\hline 2 & 0.4 & 0.5 & $-3.772 \mathrm{e}-01+7.176 \mathrm{e}-02$ & $2.046 \mathrm{e}-11$ & $3.148 \mathrm{e}-11$ \\
\hline 3 & 0.4 & 0.5 & $-5.456 \mathrm{e}-01+2.267 \mathrm{e}-01$ & $3.330 \mathrm{e}-15$ & $7.352 \mathrm{e}-15$ \\
\hline 4 & 0.4 & 0.5 & $-7.216 \mathrm{e}-01+2.988 \mathrm{e}-01$ & $7.845 \mathrm{e}-12$ & $1.855 \mathrm{e}-12$ \\
\hline 5 & 0.7 & 0.9 & $-1.147 e+00+5.787 e-02$ & $6.050 \mathrm{e}-12$ & $2.618 \mathrm{e}-11$ \\
\hline 7 & 0.6 & 0.7 & $-9.315 e-01+3.307 e-01$ & $1.006 \mathrm{e}-11$ & $3.832 \mathrm{e}-12$ \\
\hline
\end{tabular}

The coefficients $b_{l}$ and $c_{l}$ are given by

$$
\begin{aligned}
& b_{l}=\left\{l\left[1-\left(\frac{R_{1}}{R_{2}}\right)^{2}\right]\left[1-\left(\frac{W_{1}}{W_{2}}\right)^{2 l}\right]\right. \\
&\left.+\left[1-\left(\frac{R_{1}}{R_{2}}\right)^{2 l}\right]\left[\left(\frac{R_{2}}{W_{2}}\right)^{2 l}-\left(\frac{W_{1}}{R_{1}}\right)^{2 l}\right]\right\}\left[1-\left(\frac{W_{1}}{W_{2}}\right)^{2 l}\right]^{-1} \\
& c_{l}=\left\{l\left[1-\left(\frac{R_{1}}{R_{2}}\right)^{2}\right]\left[1-\left(\frac{R_{1}}{W_{2}}\right)^{2 l}\right]\left[1-\left(\frac{W_{1}}{R_{1}}\right)^{2 l}\right]\right. \\
&\left.-\left[1-\left(\frac{R_{2}}{W_{2}}\right)^{2 l}\right]\left[1-\left(\frac{W_{1}}{R_{1}}\right)^{2 l}\right]\left[1-\left(\frac{R_{1}}{R_{2}}\right)^{2 l}\right]\right\}\left[1-\left(\frac{W_{1}}{W_{2}}\right)^{2 l}\right]^{-1} .
\end{aligned}
$$

A sample of eigenvalues is shown in Table 1 for $W_{1}=1$ and $W_{2}=10$ and different aspect ratios, $d_{1}=R_{1} / W_{2}$ and $d_{2}=R_{2} / W_{2}$. The exact analytical solution, Eq. (68), is compared with the numerical solution. The relative errors in the real and imaginary parts of the eigenvalues are

$$
\begin{aligned}
& \varepsilon_{\mathrm{Re}}=\left|\frac{\operatorname{Re}(\omega)-\operatorname{Re}\left(\omega_{\text {exact }}\right)}{\operatorname{Re}\left(\omega_{\text {exact }}\right)}\right| \leq 3.2 \times 10^{-11} \\
& \varepsilon_{\operatorname{Im}}=\left|\frac{\operatorname{Im}(\omega)-\operatorname{Im}\left(\omega_{\text {exact }}\right)}{\operatorname{Im}\left(\omega_{\text {exact }}\right)}\right| \leq 1.1 \times 10^{-10} .
\end{aligned}
$$

The precision is excellent and reaches 10 digits. Our algorithm computes the eigenvalues in cylindrical geometry quickly and accurately with vacuum gaps between the plasma column and the walls. The eigenvalues obtained in this example are good initial guesses to study the relativistic problem in the low speed limit. Next, we turn to the relativistic planar case.

\subsection{Relativistic planar diode geometry}

In the relativistic planar diode geometry, the dispersion relation is also known analytically in the long wavelength limit. Thus it is an interesting case to check our algorithm for the relativistic diocotron instability. For completeness, we recall the main results. For a detailed discussion, see Davidson et al. (1987, 1988). The plasma is drifting in the $y$-direction at a speed $V_{y}$ and is located between $x=X_{1}$ and $x=X_{2}$. The cathode is located at $x=0$ and the anode at $x=d$. We use Cartesian coordinates $(x, y, z)$. The eigenvalue problem in Cartesian coordinates for the diocotron instability in a relativistic planar diode geometry is

$$
\begin{aligned}
\frac{\partial^{2} \phi}{\partial x^{2}}-k^{2} & \left(1-\frac{\omega^{2}}{k^{2} c^{2}}+\frac{\omega_{\mathrm{Dc}}^{2}}{k^{2} c^{2}}\right) \phi= \\
& -\frac{k \phi}{\omega-k V_{y}}\left(1-\frac{V_{y}}{c} \frac{\omega}{k c}\right) \frac{\partial \omega_{\mathrm{Dc}}}{\partial x}
\end{aligned}
$$

where $k$ is the wavenumber. In this paragraph, because of the Cartesian geometry, the diocotron frequency is defined as (no factor $1 / 2$ )

$\omega_{\mathrm{Dc}}=\frac{\omega_{\mathrm{p}}^{2}}{\omega_{\mathrm{c}}}$.

The applied external magnetic field is constant and uniform outside the plasma layer, $B_{z}(r)=B_{0}$ for $x \geq X_{2}$. The particle number density is chosen such that

$\frac{n(x)}{\gamma(x)}= \begin{cases}0, & W_{1} \leq x \leq X_{1} \\ \frac{n_{0}}{\gamma_{0}}=\text { const. }, & X_{1} \leq x \leq X_{2} \\ 0, & X_{2} \leq x \leq W_{2}\end{cases}$

The Lorentz factor is $\gamma=1 / \sqrt{1-V_{y}^{2} / c^{2}}$. For this particular choice of density profile, the diocotron frequency is constant throughout the layer's cross section, $\omega_{\mathrm{Dc}}=$ const. Moreover, the drift speed is

$V_{y}=c \tanh \left[\omega_{\mathrm{Dc}}\left(x-X_{1}\right) / c\right]$.

In the long-wavelength perturbation limits, corresponding to $k d \ll 1$, the dispersion relation reads

$$
\begin{aligned}
\frac{\operatorname{Re}(\omega)}{k c}= & \frac{1}{2}\left[\sinh \theta+2 \frac{\Delta_{i}}{\Delta_{b}} \theta \cosh \theta\right] \\
& \times\left[\cosh \theta+\frac{\theta}{\Delta_{b} \sinh \theta}\left(\Delta_{i} \cosh ^{2} \theta+\Delta_{0}\right)\right]^{-1} \\
\frac{\operatorname{Im}(\omega)}{k c}= & \frac{\theta}{2} \sqrt{p^{2}-\frac{\sinh ^{2} \theta}{\theta^{2}}} \\
& \times\left[\cosh \theta+\frac{\theta}{\Delta_{b} \sinh \theta}\left(\Delta_{i} \cosh ^{2} \theta+\Delta_{0}\right)\right]^{-1},
\end{aligned}
$$

where the quantities are defined by

$$
\begin{aligned}
\Delta_{i} & =X_{1} / W_{2} \\
\Delta_{b} & =\left(X_{2}-X_{1}\right) / W_{2} \\
\Delta_{0} & =\left(W_{2}-X_{2}\right) / W_{2} \\
p & =2 \sqrt{\Delta_{i} \Delta_{0}} / \Delta_{b} \\
\theta & =\omega_{\text {Dc }}\left(X_{2}-X_{1}\right) / c
\end{aligned}
$$

Results for the eigenvalues are shown in Figs. 1 and 2 in the long wavelength limit and compared with the exact dispersion relations, Eqs. (77) and (78). The relativistic diocotron instability for arbitrary wavelength is shown in Figs. 3 and 4. Our numerical results agree with very good accuracy to those found by Davidson et al. (1987, 1988), who directly solved for the dispersion relation in the general case, i.e. for arbitrary $k d$. 

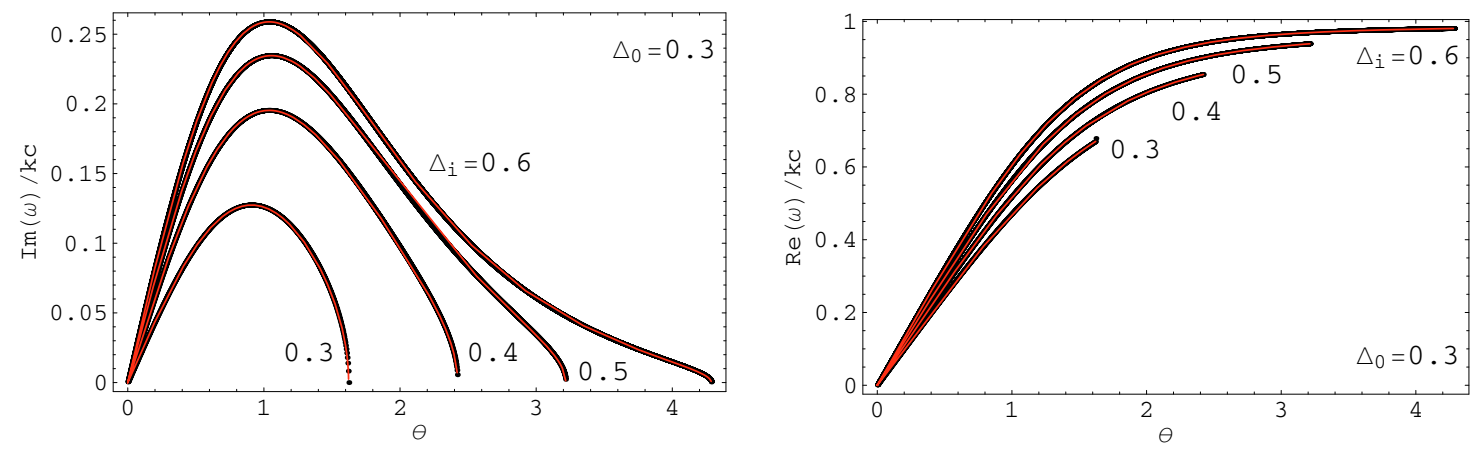

Fig. 1. Imaginary part in the left panel and real part in the right panel of the eigenfrequencies (in black dots), compared to the analytical exact dispersion relation in the long wavelength limit Eqs. (77) and (78), (red curves). The parameters are $\Delta_{0}=0.3$ and $\Delta_{i}=0.3,0.4,0.5,0.6$.
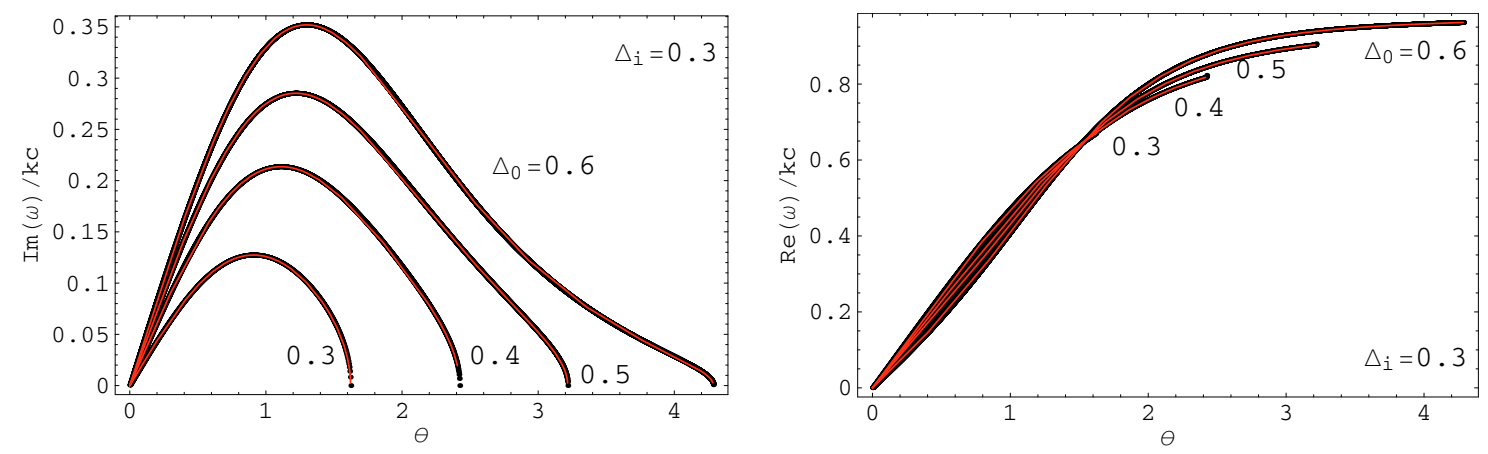

Fig. 2. Imaginary part in the left panel and real part in the right panel of the eigenfrequencies (in black dots), compared to the analytical exact dispersion relation in the long wavelength limit Eqs. (77) and (78), (red curves). The parameters are $\Delta_{i}=0.3$ and $\Delta_{0}=0.3,0.4,0.5,0.6$.
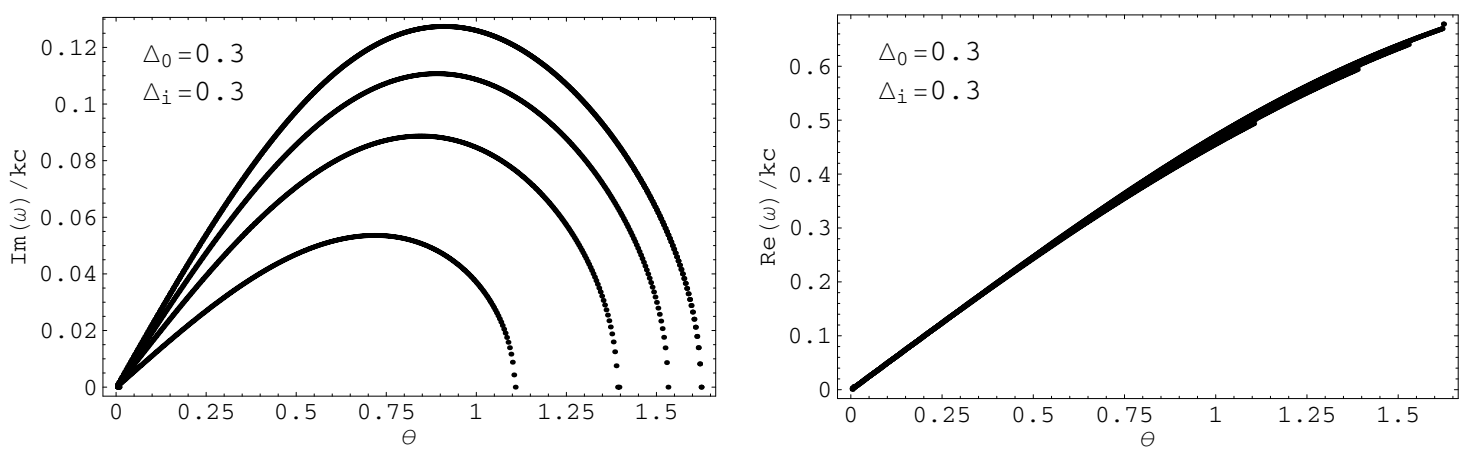

Fig. 3. Imaginary part in the left panel and real part in the right panel of the eigenfrequencies for arbitrary wavelength, $k d$. The parameters are $\Delta_{i}=0.3$ and $\Delta_{0}=0.3$. Check with Fig. 6 of Davidson et al. (1988).

\section{Results}

We have demonstrated that our numerical algorithm gives accurate results in the non-relativistic cylindrical geometry as well as in the relativistic regime in Cartesian coordinates. In this section, we compute the eigenspectra of the relativistic diocotron instability in cylindrical coordinates for various equilibrium density, electric field, and velocity profiles. Application to the pulsar's electrosphere is also discussed.

\subsection{Plasma column}

First, we consider the laboratory plasma in cylindrical geometry, confined by an external experimental electromagnetic device. The external applied magnetic field and the density profile are specified as initial data. We use the simple profile presented in Sect. 2.3.
In the non-relativistic limit, a very good first guess is given by Eq. (68). The influence of the relativistic and electromagnetic effects are then investigated by slowly increasing the maximal speed at the outer edge of the plasma column, $v_{\max }=R_{2} \Omega\left(R_{2}\right)$.

In the non-relativistic flow, the growth rate of the diocotron instability only depends on the ratios $d_{1}=R_{1} / W_{2}, d_{2}=R_{2} / W_{2}$, and $w=W_{1} / W_{2}$. In order to avoid variation in the growth rate or stabilisation of the instability purely due to geometrical effects, these ratios are kept constant, while $v_{\max }$ is increased. To this end, for instance, we increase $W_{2}$ and adjust the other parameters $R_{1}, R_{2}, W_{1}$ to fit the constant ratios conditions, $w=$ const., $d_{1}=$ const., $d_{2}=$ const.

Two cases are presented in Fig. 5. The first one has $w=0.1$, $d_{1}=0.45$, and $d_{2}=0.5$, Fig. 5a, whereas the second one has $w=0.1, d_{1}=0.4$, and $d_{2}=0.5$, Fig. 5b. The eigenvalues are conveniently normalised to the diocotron frequency $\omega_{\mathrm{D}}$. For non-relativistic speeds, $v_{\max } \ll c$, the eigenvalues of Sect. 4.1 are recovered, see Table 2 . The thinner the plasma layer, the larger 

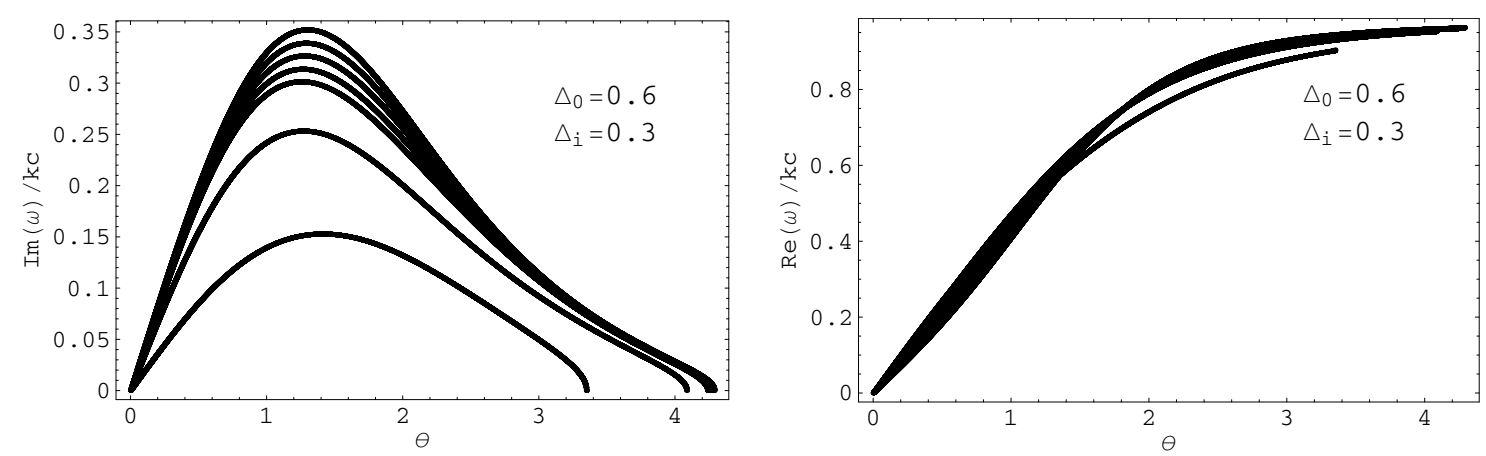

Fig. 4. Imaginary part in the left panel and real part in the right panel of the eigenfrequencies for arbitrary wavelength, $k d$. The parameters are $\Delta_{i}=0.3$ and $\Delta_{0}=0.6$. Check with Fig. 7 of Davidson et al. (1988).
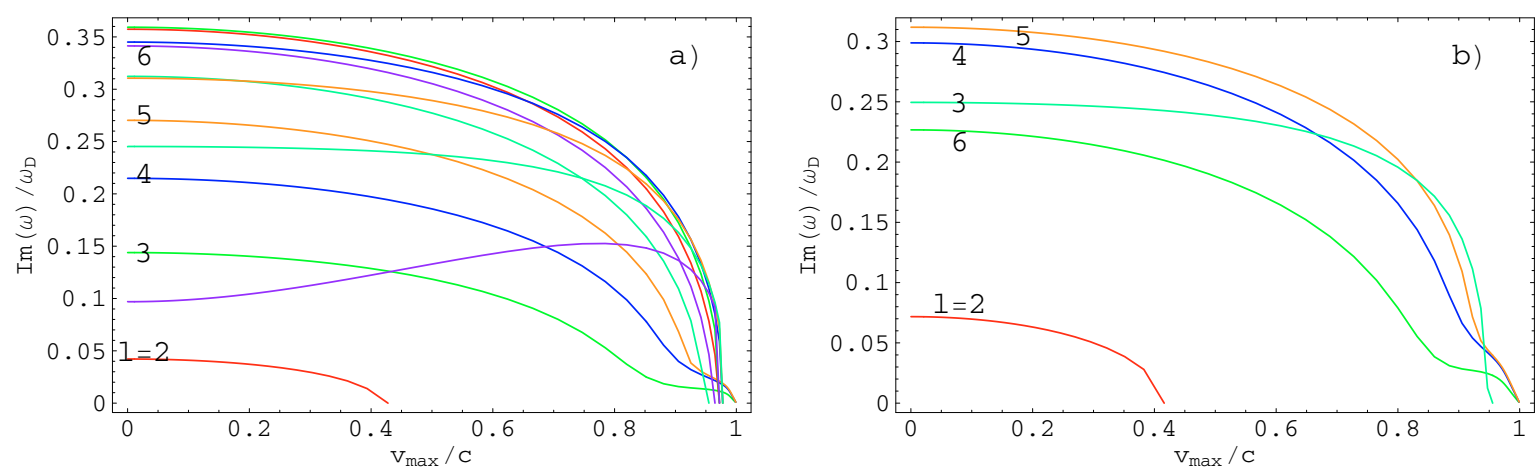

Fig. 5. Stabilisation of the diocotron instability when relativistic and electromagnetic effects are included. The relativistic diocotron frequency is constant in the whole plasma column, $\omega_{\mathrm{D}}=$ cste. The geometric aspect ratios are $w=0.1, d_{1}=0.45$, and $d_{2}=0.5$ for $\left.\mathbf{a}\right)$ and $w=0.1, d_{1}=0.4$, and $d_{2}=0.5$ for $\left.\mathbf{b}\right)$. Only the first unstable modes are labeled, $l=2,3,4,5,6$ to avoid overloading the plots. Each coloured curve depicts a different azimuthal mode number $l$.

Table 2. Eigenvalues of the plasma column for the density profile in Sect. 4.1. Comparison of the non-relativistic one $\omega_{\text {nrel }}$ and the relativistic one $\omega_{\text {rel }}$ in the low speed limit, $v_{\max } \ll c$ for $w=0.1, d_{1}=0.4$, and $d_{2}=0.5$.

\begin{tabular}{ccccc}
\hline \hline mode $l$ & $\omega_{\text {nrel }}$ & $\omega_{\text {rel }}$ & \\
\hline 2 & $3.772986 \mathrm{e}-01+7.176435 \mathrm{e}-02$ & $\mathrm{i}$ & $3.773015 \mathrm{e}-01+7.174847 \mathrm{e}-02$ & $\mathrm{i}$ \\
3 & $5.456744 \mathrm{e}-01+2.267241 \mathrm{e}-01$ & $\mathrm{i}$ & $5.456773 \mathrm{e}-01+2.267196 \mathrm{e}-01$ & $\mathrm{i}$ \\
4 & $7.216191 \mathrm{e}-01+2.988911 \mathrm{e}-01$ & $\mathrm{i}$ & $7.216219 \mathrm{e}-01+2.988890 \mathrm{e}-01$ & $\mathrm{i}$ \\
5 & $9.004354 \mathrm{e}-01+3.118851 \mathrm{e}-01$ & $\mathrm{i}$ & $9.004382 \mathrm{e}-01+3.118852 \mathrm{e}-01$ & $\mathrm{i}$ \\
6 & $1.080114 \mathrm{e}+00+2.495296 \mathrm{e}-01$ & $\mathrm{i}$ & $1.080116 \mathrm{e}+00+2.495328 \mathrm{e}-01$ & $\mathrm{i}$ \\
\hline
\end{tabular}

the number of unstable modes, 5 and 12 unstable modes, respectively. In both cases, the growth rate starts to be altered whenever $v_{\max } / c \gtrsim 0.1$. We only labeled the first 5 unstable modes $l=2,3,4,5,6$ in order to avoid overloading the figure.

In any case, for very high speeds, $v_{\max } / c \approx 1$, all the diocotron modes become stable because the growth rate vanishes (Fig. 5). The stabilisation process already observed in the relativistic planar diode is not altered by the cylindrical geometry.

\subsection{Relativistic planar diode limit}

The influence of the curvature is also studied by taking the limit of the planar diode geometry. The curvature of the plasma column is then increased to investigate the evolution of the growth rates.

The effective aspect ratio of the plasma layer is conveniently described by the parameter

$A=\frac{R_{1}}{R_{2}-R_{1}}$
Using the equilibrium electric field profile indicated in Sect. 2.4, in the limit of small curvature corresponding to a large aspect ratio, $A \rightarrow+\infty$, the eigenvalue problem and equilibrium configuration are described by the relativistic planar diode.

We show the evolution of the growth rate in the non relativistic limit, $v_{\max }=10^{-3}$, Fig. $6 \mathrm{a}$, and in the relativistic case, $v_{\max }=0.3$, Fig. $6 \mathrm{~b}$. The eigenvalues are conveniently normalised to the value of the diocotron frequency at the outer boundary of the plasma column, $\omega_{\mathrm{D}}\left(R_{2}\right)$. The aspect ratio has a drastic influence on the growth rate. For high values of $A \gg 1$, all unstable modes are stabilised, in both non-relativistic and relativistic flows. Note, however, that in the relativistic regime, the modes $l=2$ and $l=3$ have already been stabilised, whatever the aspect ratio.

When the aspect ratio $A$ is increased, the fastest growing unstable mode shifts to higher azimuthal numbers $l$, whereas the lower azimuthal numbers $l$ start to stabilise. Indeed, to compute the eigenvalues for different aspect ratios, we increase $R_{1}$ and $R_{2}$ while keeping the difference $\Delta=R_{2}-R_{1}$ constant. Assuming that the geometrical size of the perturbation fits into the layer 

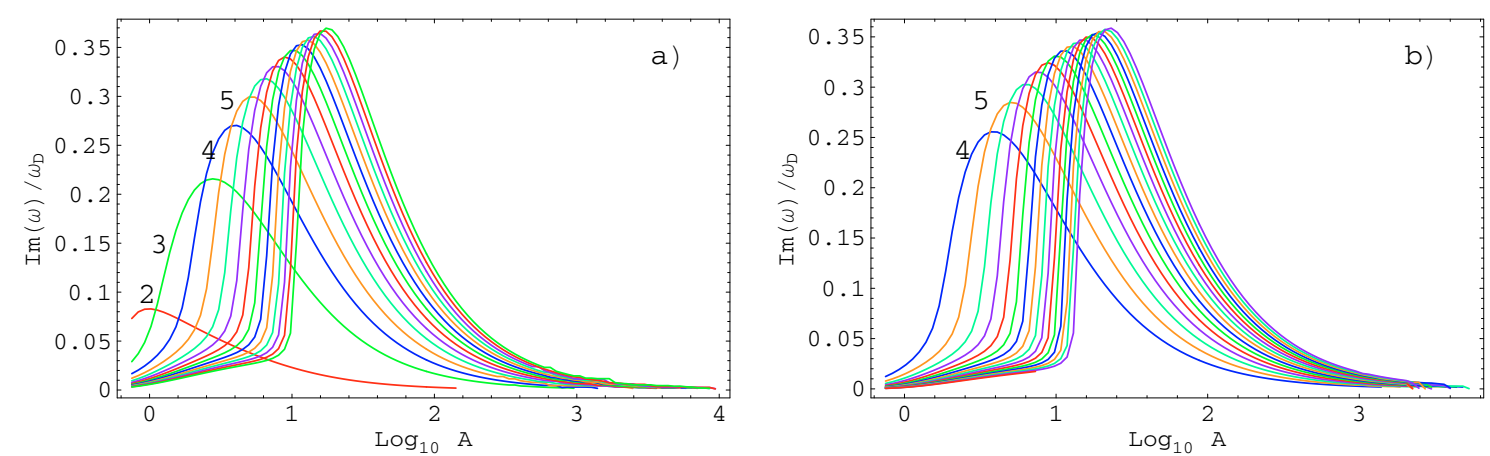

Fig. 6. Effect of the cylindrical geometry on the growth rate of the relativistic diocotron instability. The growth rate is normalised to the diocotron frequency and plotted versus the aspect ratio $A$. Non-relativistic regime, $v_{\max }=10^{-3}$ in a) and relativistic speed, $v_{\max }=0.3$ in b). Only the first unstable modes are labeled, $l=2,3,4,5$ to avoid overloading the plots. Each coloured curve depicts a different azimuthal mode number $l$.

Table 3. Parameters for the three rotation profiles used to mimic the azimuthal velocity of the plasma in the electrospheric disk.

\begin{tabular}{cccc}
\hline \hline$\Omega$ & $\alpha$ & $\beta$ & $r_{0}$ \\
\hline$\Omega_{1}$ & 3.0 & $5 \times 10^{-5}$ & 6.0 \\
$\Omega_{2}$ & 1.0 & $5 \times 10^{-5}$ & 6.0 \\
$\Omega_{3}$ & 0.3 & $5 \times 10^{-5}$ & 10.0 \\
\hline
\end{tabular}

thickness $\Delta$, we get an estimate of the allowed azimuthal numbers such that

$\frac{2 \pi}{l} R_{1} \approx \Delta$.

Therefore the fastest mode numbers are roughly $l \propto A$ and linearly growing with the aspect ratio.

\subsection{Electrosphere}

The electrospheric non-neutral plasma, as already proved in previous works by Krause-Polstorff \& Michel (1985a) and Pétri et al. (2002b), is confined by the rotating magnetised neutron star. The most important feature is the velocity profile in the plasma column. For simplicity, here, we assume that no vacuum gaps exist between the plasma and the walls, so that $W_{1}=R_{1}$ and $W_{2}=R_{2}$. The rotation profile is chosen to mimic the rotation curve obtained in the 3D electrosphere. To study the influence of the relativistic effects, we take the same profiles as those given in Pétri (2007). We recall that three different analytical expressions for the radial dependence of $\Omega$ are chosen by mainly varying the gradient in differential shear, as follows

$\Omega(r)=\Omega_{*}\left(2+\tanh \left[\alpha\left(r-r_{0}\right)\right] \mathrm{e}^{-\beta r^{4}}\right)$.

The values used are listed in Table 3 . The angular velocity starts from corotation with the star $\Omega=\Omega_{*}$, followed by a sharp increase around $r=6$ for $\Omega_{1,2}$, and a less pronounced gradient around $r=10$ for $\Omega_{3}$. Finally the rotation rate asymptotes twice the neutron star rotation speed (Fig. 7). The results of Krause-Polstorff \& Michel (1985a) and Pétri et al. (2002b) have been computed for non-relativistic speeds. However, confinement of non-neutral plasmas by some external electromagnetic fields is very general and also applies when relativistic effects are taken into account (Tsang \& Davidson 1986). In the case of relativistic motions within the electrosphere, we would expect some quantitative changes in the rotation curves because of the relativistic factor appearing in the expression for the charge density (due to the current displacement):

$\rho_{\mathrm{e}}=-2 \varepsilon_{0} \frac{\boldsymbol{\Omega} \cdot \boldsymbol{B}}{1-r^{2} \Omega^{2} / c^{2}}$.

Nevertheless, differential rotation is still expected, even in the latter case. That is why we took typical rotation curves like those depicted in Fig. 7a. We emphasise that the electromagnetic field, as well as the charge density resulting from the chosen rotation profiles, are determined in a fully self-consistent manner, as described in Sect. 2. Relativistic speeds are only reached in the extended part of the electrosphere, i.e. the outer part approaching the light cylinder. In Fig. 7b, the linear speed in the disk is plotted as $r \Omega(r) / v_{\max }$, and the highest speed $v_{\max }<c$ corresponds to the largest radii $r$, i.e. the outer part of the disk. Nevertheless, in the region where strong gradient exists, around $r=6$, the denominator of Eq. (87) remains close to unity. Indeed, in the differentially rotating part, we have $r \Omega(r) / v_{\max } \lesssim 1 / 2$, implying $\left(1-r^{2} \Omega^{2} / c^{2}\right)^{-1} \lesssim 4 / 3$, therefore the non-relativistic calculations are still valid in this part of the disk with an error less than roughly $20 \%$. Actually, the exact rotation profile is not important in our study, since we just want to demonstrate that the diocotron instability still exists in the relativistic regime.

We generalised the study presented in Pétri (2007) by including the relativistic effects, i.e. relativistic speed of the flow and electromagnetic field perturbation. We started with a nonrelativistic rotation profile such that $\Omega_{*} R_{2} \ll c$ and slowly increased $R_{2}$ (as well as $W_{1}, W_{2}, R_{1}$ to maintain their ratio constant) in order to approach the speed of light for the maximal rotation rate of the plasma column. First, we computed the growth rates when an outer wall is presented. Second, we remove this wall, imposing outgoing wave solutions, and finally compared both situations.

\subsubsection{Outer wall}

To remain fully self-consistent, we only consider an uniform applied external magnetic field. The maximum growth rates, normalised to the angular velocity of the neutron star, for the first rotation curve, $\Omega_{1}$, for each mode $l$, is shown in Fig. 8a) for an extension from $R_{1}=1$ to $R_{2}=20$. Each coloured curve depicts a different azimuthal mode number $l$. However, they are not labeled, because we want only to demonstrate the stabilisation of all modes. The precise value of the eigenvalues associated with a particular mode $l$ therefore does not matter in our present study. The most important characteristic is the behaviour of the full set of unstable eigenvalues when the system becomes relativistic. 

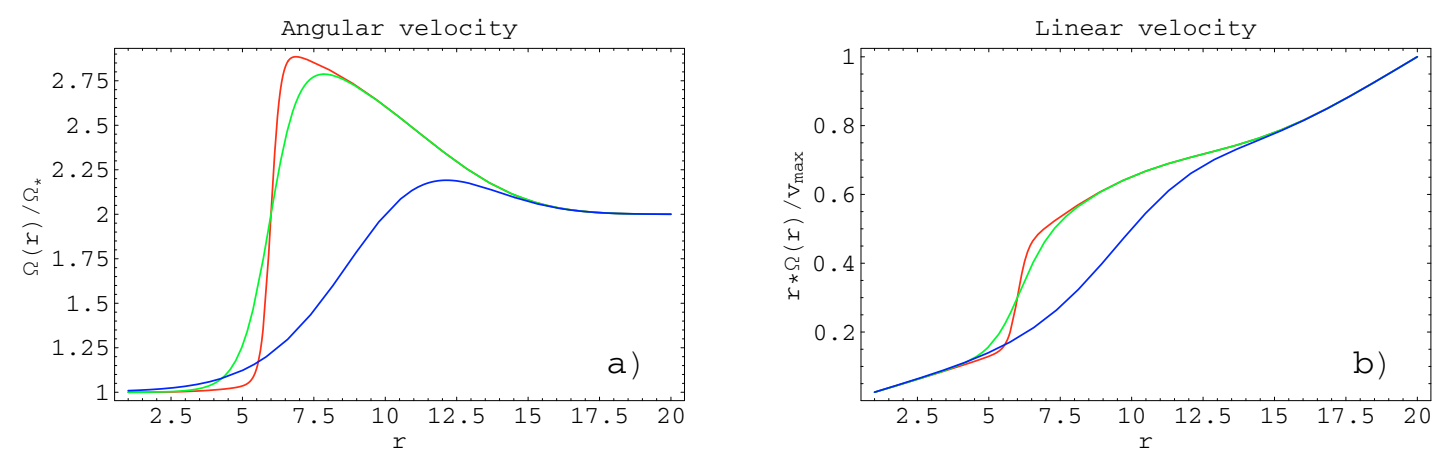

Fig. 7. Three choices of differential rotation curves in the plasma column for the cylindrical pulsar electrosphere, $\Omega_{1}$ in red, $\Omega_{2}$ in green, and $\Omega_{3}$ in blue, a). The linear speed is depicted in $\mathbf{b})$ in units of $v_{\max }=R_{2} \Omega\left(R_{2}\right)$.
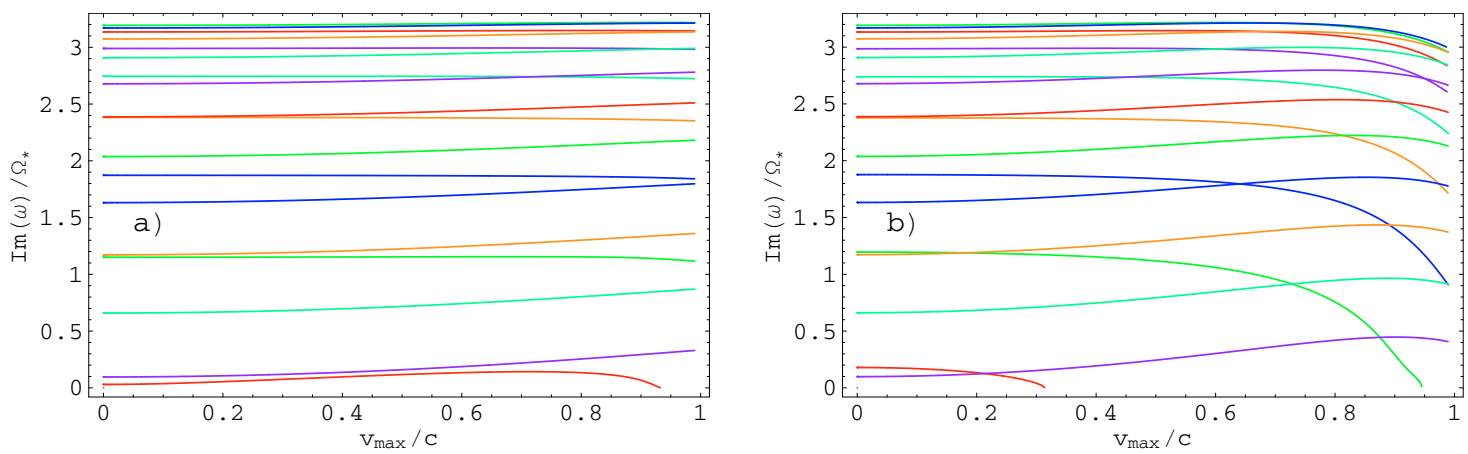

Fig. 8. Evolution of the growth rates $\operatorname{Im}(\omega)$ of the profiles $\Omega_{1}$ for increasing maximal speed of the column. The column extends from $R_{1}=1$ to $R_{2}=20$ in a), and from $R_{1}=1$ to $R_{2}=10$ in b). Each coloured curve depicts a different azimuthal mode number $l$, but they are not labeled. The outer wall is located at $R_{2}$.
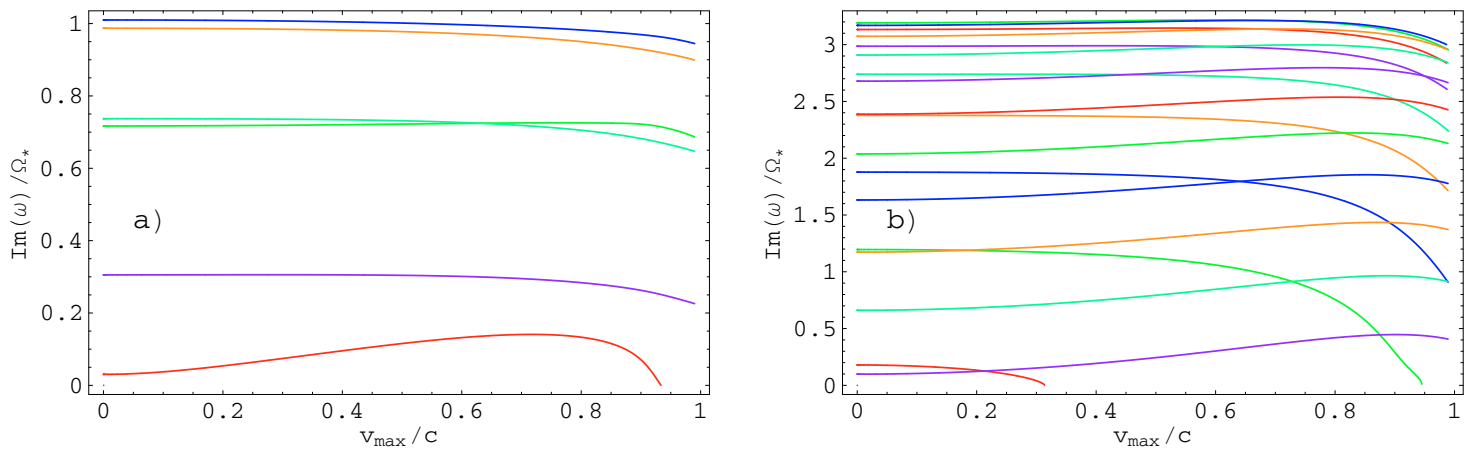

Fig. 9. Evolution of the growth rates $\operatorname{Im}(\omega)$ of the profiles $\Omega_{2}$ for increasing maximal speed of the column. The column extends from $R_{1}=1$ to $R_{2}=20$ in $\mathbf{a}$ ), and from $R_{1}=1$ to $R_{2}=10 \mathrm{in} \mathbf{b}$ ). The outer wall is located at $R_{2}$.

The profile with the steepest gradient possesses the largest number of excited unstable modes because it corresponds to the case where the smallest scales appear, i.e. $l$ large. In the nonrelativistic limit, $v_{\max } \ll c$, the highest growth rate, for $l=8$ has a value of $\gamma_{\max }=3.2$. The spectrum is exactly the same as in Pétri (2007). When the maximal speed is increased, the growth rates vary significantly, but we do not observe the stabilisation effect except for the mode $l=1$, which disappears for $v_{\max }=0.936$. For relativistic speed, $v_{\max } \lesssim c$, the other modes roughly keep their growth rate at their value for the nonrelativistic instability. Several of them even increase.

In order to demonstrate the tendency towards stabilisation in the electrosphere, we reduce the size of the plasma column. For instance, we choose the plasma extension from $R_{1}=1$ to $R_{2}=$ 10. The new growth rates are shown in Fig. 8b. The diocotron instability now tends to stabilise for all modes $l$. Close to the speed of light, the growth rates start to decrease significantly. Note that the $l=1,2$ modes have already disappeared (Fig. 8b).
The second steepest profile possesses stabler modes than we would expect due to the fact that only large scale structures can emerge with this slope of the differential rotation (Fig. 9a). Here, the tendency to stabilise the diocotron instability is more evident. The mode $l=1$ disappears as in the previous case. The other modes begin to show a significant decrease in their growth rate when approaching the ultra-relativistic limit. Here again, we checked that for low speeds, we recover the non-relativistic spectrum with good accuracy. For the narrower layer, the stabilisation is fully achieved for all the modes (Fig. 9b).

Finally, the third smooth profile has only four unstable modes (Fig. 10a). The stabilisation effects are clearly seen for the modes $l=2$, whereas the other modes are near to full stabilisation. Reducing the size of the electrosphere here, we again observe full disappearance of the diocotron instability (Fig. 10b).

The proof of the relativistic stabilisation effect is therefore obvious for the profiles $\Omega_{2}$ and $\Omega_{3}$. It is clearly seen that the diocotron instability is suppressed when the maximal speed 

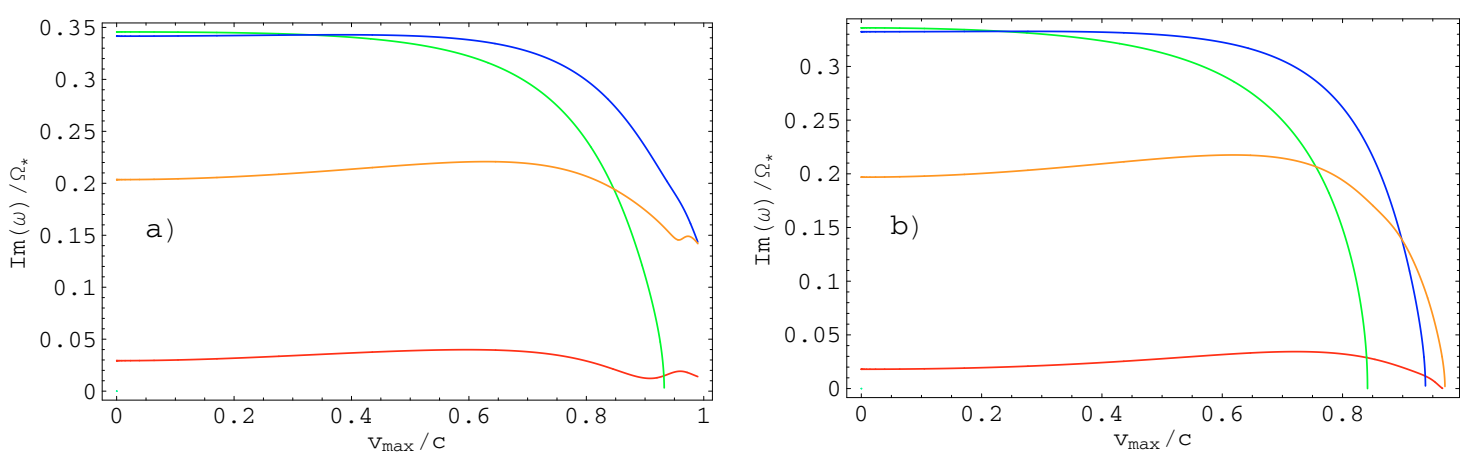

Fig. 10. Evolution of the growth rates $\operatorname{Im}(\omega)$ of the profiles $\Omega_{3}$ for increasing maximal speed of the column. The column extends from $R_{1}=1$ to $R_{2}=20 \mathrm{in} \mathbf{a}$ ), and from $R_{1}=1$ to $\left.R_{2}=15 \mathrm{in} \mathbf{b}\right)$. The outer wall is located at $R_{2}$.
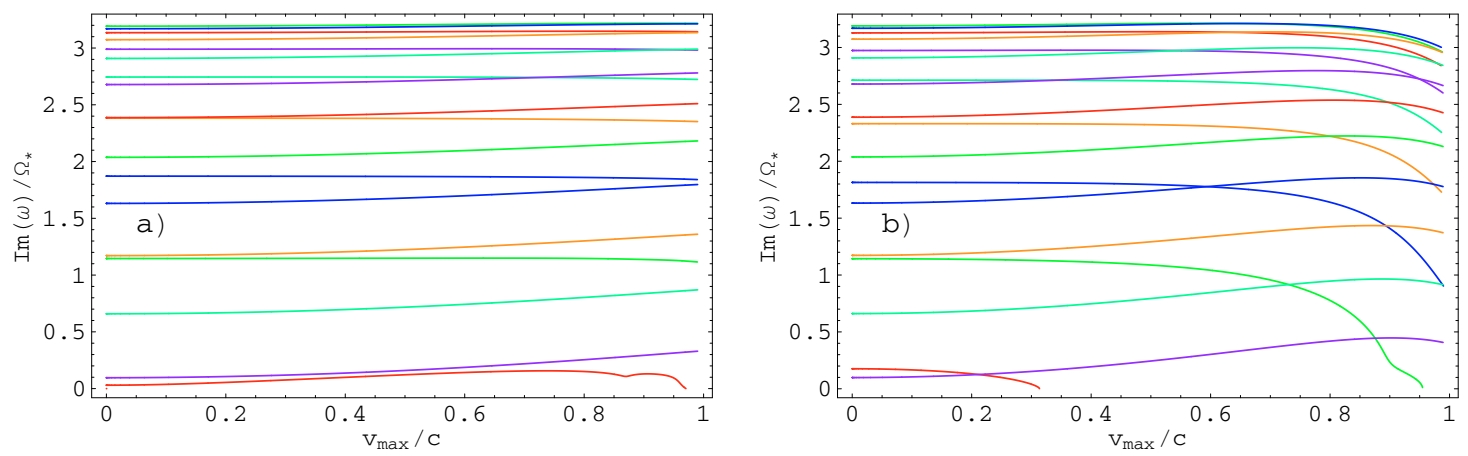

Fig. 11. Same as Fig. 8, but outgoing-wave boundary conditions are applied.
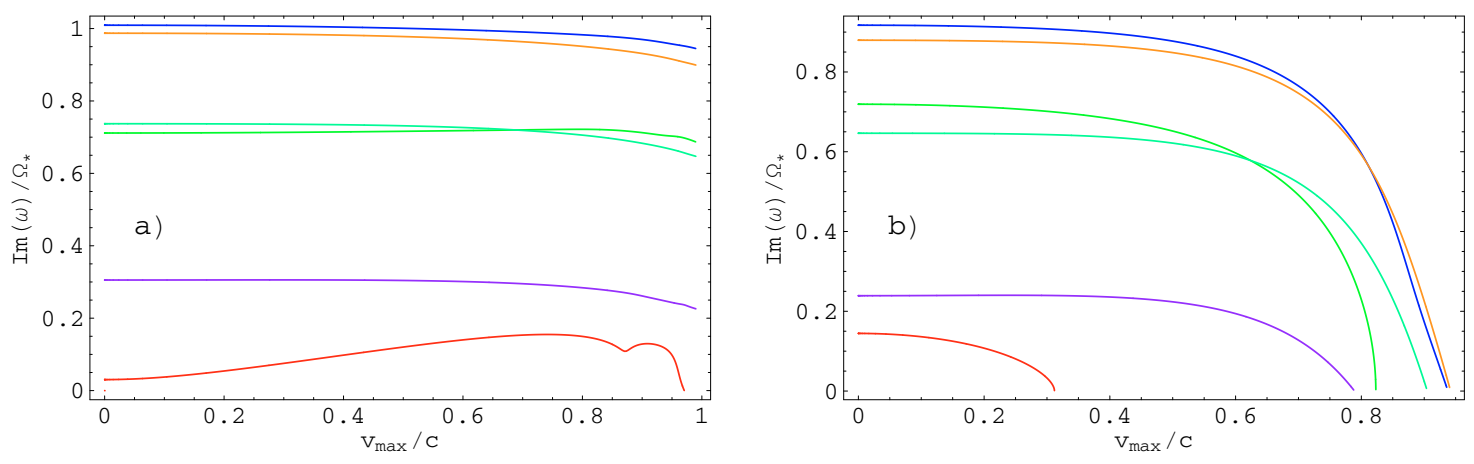

Fig. 12. Same as Fig. 9, but outgoing-wave boundary conditions are applied.

approaches the speed of light with a steep decrease in the growth rate for $v_{\max } \lesssim c$. These examples undoubtedly reveal the influence of relativistic and electromagnetic effects towards stabilisation of the non-neutral plasma in the pulsar electrosphere.

To understand the change in behaviour of the diocotron instability when simply changing the size of the plasma column or, equivalently, the dimension of the electrosphere, we need to understand how this instability is generated. The instability is launched from the resonance between the motion of the wave pattern related to the perturbation and particles in the flow. It is readily seen from the denominator of Eq. (54). The corotation radius $r_{\mathrm{c}}$ satisfies

$\operatorname{Re}(\omega)=l \Omega\left(r_{\mathrm{c}}\right)$.

In order for the relativistic effects to play a significant role, the speed in the vicinity of the corotation region should be close to the speed of light. If the tail of the rotation curve is long, the outer edge of the plasma attains speeds close to $c$, while the speed near the corotation radius remains non-relativistic. Thus, the growth rates are not significantly affected by the relativistic effects. However, reducing the tail of the rotation curve allows the region near corotation to reach higher velocities. Therefore, the stabilisation starts to set in and the diocotron instability can be suppressed.

\subsubsection{Outgoing waves}

We perform a second set of calculations by removing the outer wall assumption and enforcing outgoing electromagnetic waves propagation into vacuum. The boundary condition on the outer plasma/vacuum interface has been discussed in Sect. 3.3.

We use exactly the same configurations and rotation profiles presented in the previous section. The only change comes from the outer boundary condition, namely $\phi_{\mathrm{III}}\left(W_{2}\right)=0$ which is replaced by Eq. (64). The results are shown in Figs. 11-13 for the rotation curve $\Omega_{1}, \Omega_{2}$, and $\Omega_{3}$, respectively. Comparing both situations, the growth rates are not significantly affected by wave emission. Note, however, that, because energy is carried away by Poynting flux, the instability grows more slowly than in the previous case. 

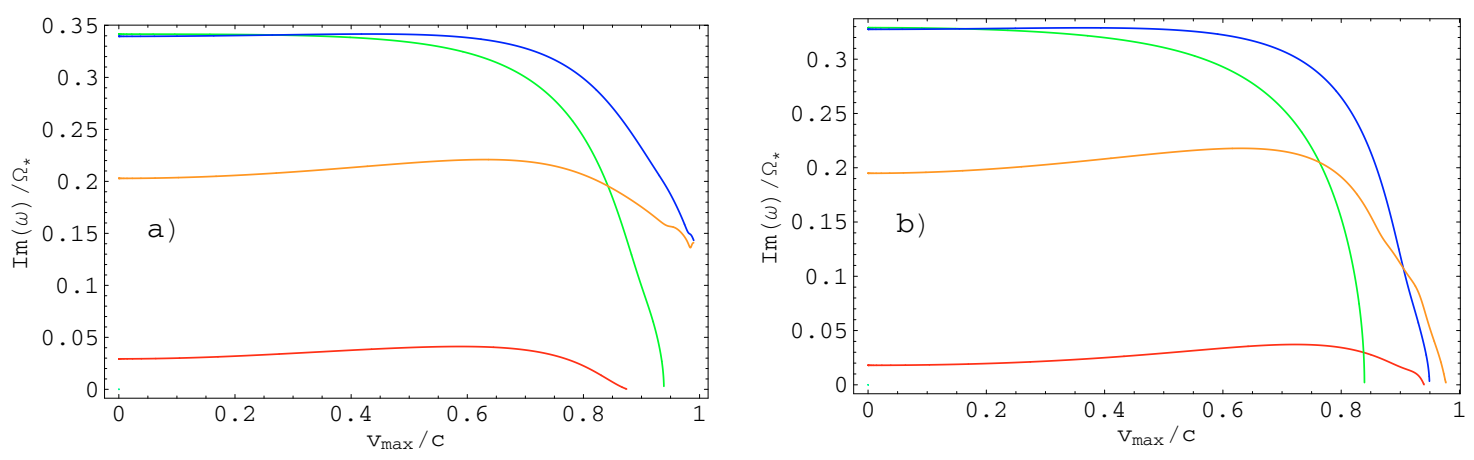

Fig. 13. Same as Fig. 10, but outgoing-wave boundary conditions are applied.

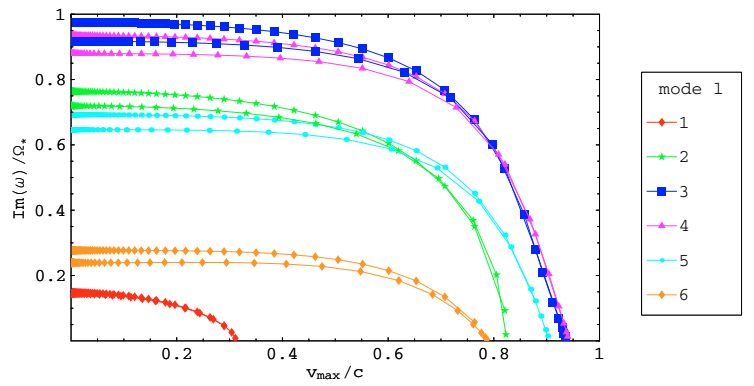

Fig. 14. Comparison of the outer wall and outgoing wave boundary conditions for the profile $\Omega_{2}$, Figs. $9 \mathrm{~b}$ and $12 \mathrm{~b}$. The growth rates for outgoing waves are almost always slightly lower.

In Fig. 14, we show a comparison between both boundary conditions for the profile $\Omega_{2}$. It is clearly seen that the growth rates are relatively insensitive to the nature of the boundary. Nevertheless, there is a tendency to decrease the growth rates when outgoing waves are present.

\section{Conclusion}

We developed a numerical code to compute the eigenspectra and eigenfunctions of the diocotron instability, including electromagnetic and relativistic effects. In the non-relativistic limit, we recovered and confirmed the results obtained by another technique using pseudo-spectral method by the Chebyshev expansion (Pétri 2007). Unstable modes are computed for a uniform external applied magnetic field and arbitrary velocity, density, and electric field profiles. Application to a plasma column, as well as to the pulsar electrosphere, has been shown. In both cases, the diocotron regime gives rise to instabilities with decreasing growth rates when the maximal speed of the flow becomes relativistic. While the growth rates can be comparable to the rotation period of the neutron star in the nonrelativistic limit, it is found that, for special rotation profiles, the diocotron instability is completely suppressed in the relativistic regime. Including electromagnetic wave emission from the electrospheric plasma does not change these conclusions drastically.

What therefore happens to the plasma in the vicinity of the light cylinder needs a more general treatment including inertia of the particles, because the plasma kinetic energy becomes comparable to the magnetic field energy density. At the light cylinder, the plasma will therefore be subject to the magnetron instability.
It is the most general case, including relativistic flow, electromagnetic perturbation, and inertia of the particles. The study of the magnetron instability in a pulsar electrosphere is the aim of a forthcoming paper.

Last but not least, the influence of finite temperature in the plasma on the diocotron or magnetron instability would require a kinetic treatment of the stability via the Vlasov-Maxwell equation. This is also left for future work.

Acknowledgements. I am grateful to Jean Heyvaerts and John Kirk for helpful suggestions and comments. This work was supported by a grant from the G.I.F., the German-Israeli Foundation for Scientific Research and Development.

\section{References}

Abramowitz, M., \& Stegun, I. A. 1965, Handbook of mathematical functions with formulas, graphs, and mathematical tables, Dover Books on Advanced Mathematics (New York: Dover), Corrected edition, ed. M. Abramowitz, \& I. A. Stegun

Ayres, V. M., Chen, H. C., Stark, R. A., Uhm, H. S., \& Brandt, H. E. 1992, Physics of Fluids B, 4, 3396

Davidson, R. C. 1990, Physics of non neutral plasmas (Addison-Wesley Publishing Company)

Davidson, R. C., Tsang, K. T., \& Uhm, H. S. 1987, Phys. Lett. A, 125, 61

Davidson, R. C., Tsang, K. T., \& Uhm, H. S. 1988, Phys. Fluids, 31, 1727

Kato, S. 1989, PASJ, 41, 745

Krause-Polstorff, J., \& Michel, F. C. 1985a, MNRAS, 213, 43P

Krause-Polstorff, J., \& Michel, F. C. 1985b, A\&A, 144, 72

Michel, F. C. 2005, in Rev. Mex. Astron. Astrofis. Conf. Ser., 27

Morse, P. M., \& Feshbach, H. 1953, Methods of theoretical physics, International Series in Pure and Applied Physics (New York: McGraw-Hill) Neukirch, T. 1993, A\&A, 274, 319

Nowak, M. A., \& Wagoner, R. V. 1991, ApJ, 378, 656

Okazaki, A. T., Kato, S., \& Fukue, J. 1987, PASJ, 39, 457

Oneil, T. M. 1980, Phys. Fluids, 23, 2216

O'Neil, T. M., \& Smith, R. A. 1992, Physics of Fluids B, 4, 2720

Pasquini, T., \& Fajans, J. 2002, in Non-Neutral Plasma Physics IV, ed. F. Anderegg, C. F. Driscoll, \& L. Schweikhard, AIP Conf. Proc., 606, 453

Perez, C. A., Silbergleit, A. S., Wagoner, R. V., \& Lehr, D. E. 1997, ApJ, 476, 589

Pétri, J. 2007, A\&A, 464, 135

Pétri, J., Heyvaerts, J., \& Bonazzola, S. 2002a, A\&A, 387, 520

Pétri, J., Heyvaerts, J., \& Bonazzola, S. 2002b, A\&A, 384, 414

Pétri, J., Heyvaerts, J., \& Bonazzola, S. 2003, A\&A, 411, 203

Rylov, I. A. 1989, Ap\&SS, 158, 297

Shibata, S. 1989, Ap\&SS, 161, 187

Smith, I. A., Michel, F. C., \& Thacker, P. D. 2001, MNRAS, 322, 209

Spitkovsky, A., \& Arons, J. 2002, in Neutron Stars in Supernova Remnants, ed. P. O. Slane, \& B. M. Gaensler, ASP Conf. Ser., 271, 81

Stratton, J. A. 1941, Electromagnetic Theory (New York: McGraw-Hill)

Thielheim, K. O., \& Wolfsteller, H. 1994, ApJ, 431, 718

Tsang, K. T., \& Davidson, R. C. 1986, Phys. Rev. A, 33, 4284

Zachariades, H. A. 1993, A\&A, 268, 705 